Cahiers de recherches médiévales

\title{
La Figure de César dans les recueils biographiques de la Renaissance
}

Daniel Ménager

\section{(2) OpenEdition \\ Journals}

Édition électronique

URL : https://journals.openedition.org/crm/844

DOI : $10.4000 / \mathrm{crm} .844$

ISSN : 1955-2424

Éditeur

Honoré Champion

Édition imprimée

Date de publication : 30 mars 2006

Pagination : $9-21$

ISSN : $1272-9752$

Référence électronique

Daniel Ménager, «La Figure de César dans les recueils biographiques de la Renaissance », Cahiers de recherches médiévales [En ligne], 13 spécial | 2006, mis en ligne le 03 avril 2009, consulté le 15 décembre 2022. URL : http://journals.openedition.org/crm/844 ; DOI : https://doi.org/10.4000/crm 844 


\section{酷M}

\section{La Figure de César dans les recueils biographiques de la Renaissance}

Le propre des grandes figures mythiques, on le sait bien, est de ne jamais épuiser le commentaire. Quand on croit que tout est dit, elles reviennent nous interroger, nous intriguer. Il en va ainsi de certains empereurs, des conquérants, de Jules César. En 1981, un beau colloque réunissait à la Sorbonne des spécialistes de l'antiquité, du Moyen Âge, de la Renaissance, et des siècles suivants. Le sujet n'était autre que la présence de César dans la culture occidentale ${ }^{1}$. Dans cet ensemble, pas moins de dix communications étaient consacrées à la Renaissance, toutes de belle facture. Et pourtant, il reste à dire sur la figure de César à cette époque. Nos prédécesseurs avaient laissé de côté les recueils biographiques. Or on sait bien, surtout depuis le livre de Patricia Eichel-Lojkine ${ }^{2}$, que, sous des formes variées, les recueils en question, héritiers de Plutarque et de Suétone, sont les signes et les moyens d'un culte des grands hommes. Ce qu'ils disent de César s'inscrit dans cette perspective. J'adopte ici le point de vue de Jakob Burckhardt ${ }^{3}$, un peu oublié à notre époque, ce qui me semble injuste. S'il est une figure historique qui exalte la puissance de l'individu tout en lui laissant une part de mystère, c'est bien César que l'on peut donc envisager dans l'optique du grand historien suisse. Deux surprises cependant m'étaient réservées. D'abord, je ne trouvais pas César là où je l'attendais. Pas trace de lui dans l'un des recueils biographiques les plus connus de la Renaissance, celui de Paul Jove ${ }^{4}$, alors qu' on y rencontre Alexandre, Pyrrhus, Hannibal, Scipion l'Africain et bien d'autres. À l'heure qu'il est, je ne m'explique toujours pas les raisons de cette absence. D'autre part, l'une des grandes vies de César ne date pas du $\mathrm{XVI}^{\mathrm{e}}$ siècle, ni même du XV $\mathrm{XV}^{\mathrm{e}}$ siècle, mais du $\mathrm{XIV}^{\mathrm{e}}$ siècle : je veux parler du De gestis Cesaris de Pétrarque, texte étonnant, qui devait être simplement l'un des chapitres du De viris illustribus mais qui a peu à peu pris une telle autonomie qu'on peut le considérer à part ${ }^{5}$. Si l'on en croit la légende, l'auteur a travaillé à ce livre jusqu'à sa mort, il y a jeté ses dernières forces, ce qui n'a pas laissé d'intriguer les spécialistes de son œuvre. Bien que je ne fasse pas partie de ceux-ci, et parce que la Renaissance commence avec Pétrarque, je ne l'ai pas laissé de côté, et c'est par lui que je terminerai. Auparavant, je tenterai d'expliquer dans quel contexte idéologique la figure de César opère une sorte de percée, et comment le mystère de sa personnalité éclipse dans une large mesure les accusations de tyrannie.

${ }^{1}$ Présence de César, Paris, Les Belles-lettres, 1985.

${ }^{2}$ Le Siècle des grands hommes, éditions Peeters, 2001.

${ }^{3}$ Bien entendu dans la Civilisation de la Renaissance en Italie, qui, à mon avis, reste un chef d'œuvre. Que quelques informations doivent être rectifiées, c'est certain. Mais la thèse centrale tient toujours, et l'on ne peut qu'admirer la capacité de synthèse de l'auteur.

${ }^{4}$ Paolo Giovio, Elogia virorum bellica virtute illustrium, édition Perna, Bâle, 1575, BNF, Inv. G 1161. Sur ce recueil, voir P. Eichel-Lojkine, op. cit., p. 105-137 et 159-212.

${ }^{5}$ Voir la bibliographie sur cette œuvre, infra, n. 38.

Cahiers de Recherches Médiévales, 13spé, 2006 
Pour parler de César, on possède à la Renaissance une abondance de textes. À tout seigneur tout honneur: commençons par Plutarque. Inutile de redire l'influence des Vies parallèles sur les auteurs de cette époque, avant même qu'Amyot ne les traduise. Rappelons quand même que c'est la traduction d'Amyot qui a été traduite en anglais par Thomas North et que cette traduction, «fameuse entre toutes par son style magnifique $»^{6}$ a été celle dont s'est servi Shakespeare pour plusieurs pièces de son théâtre. Quant à Suétone, chacun sait qu'il a été fréquemment édité, commenté et même traduit au $\mathrm{XVI}^{\mathrm{e}}$ et au début du XVII ${ }^{\mathrm{e}}$ siècle ${ }^{7}$. On aurait tort de croire, cependant, que les biographies de la Renaissance puisent seulement à cette double source. Il faudrait mentionner encore les Faits et dits de Valère Maxime, l'œuvre de César lui-même et celle de Cicéron. On le comprendra mieux quand on abordera la biographie de Pétrarque. Revenons aux Vies de Plutarque. L'une de ses distinctions les plus précieuses concerne la différence entre historia et bios. Elle se trouve en particulier dans la Vie d'Alexandre ${ }^{8}$. D'un côté, celui de l'histoire, il y a «les faits et adventures des hommes»; de l'autre, «leur nature, leurs dicts et leurs moeurs». Laissons de côté le fait que l'évêque d'Auxerre, auquel j'emprunte les termes que je viens de citer, modifie un peu la pensée de l'auteur grec. Il reste l'idée, capitale, que la Vie ou la biographie cherche, à travers de multiples circonstances, y compris les détails les plus minuscules, à s'approcher de la psychologie des grands hommes. C'est d'ailleurs ce qui fascinait Montaigne chez Plutarque. Dans cette tâche, la rhétorique offre des instruments qui ne sont pas à dédaigner. La figure de César, comme celle des autres héros de Plutarque, entre dans un parallèle, forme diptyque avec celle d'Alexandre, ce qui est aussi une manière de mieux le comprendre. C'est le lecteur qui est invité à l'établir puisque celui que Plutarque avait tracé en bonne et due forme ne nous est pas parvenu'. César et Alexandre: quoi de plus naturel? Les deux plus grands conquérants de l'histoire ancienne se façonnent l'un par rapport à l'autre. La comparaison est d'autant plus pertinente que le second professait la plus grande admiration pour le fondateur de l'empire macédonien ${ }^{10}$. En outre, César se présentait comme le descendant de Vénus tout comme Alexandre se tenait comme celui d'Héraklès et d'Achille ${ }^{11}$.

Presque tout ce qui a été écrit à la Renaissance sur Jules César se trouve déjà chez Suétone et Plutarque, surtout chez le second. Les maux de tête et le «mal ca-

\footnotetext{
${ }^{6}$ Henri Fluchère, in Shakespeare, CEuvres complètes, Paris, Gallimard, «La Pléiade », t. II, p. LXXXII.

${ }^{7}$ L'une des éditions les plus importantes est celle de Philippe Béroalde (1510-1512), assortie d'un commentaire. Une édition de 1610 rassemble les commentaires latins de Béroalde, Egnatius, Érasme (liste de loca) et Glareanus. Ce dernier commentaire, qui concerne le De bello Gallico, a été étudié par J.-Cl. Margolin dans Présence de César, op. cit., p. 183-212. Montaigne possédait un exemplaire de l'édition de Béroalde: voir le Dictionnaire de Michel de Montaigne, sous la direction de Philippe Desan, Paris, Champion, 2004, p. 949. Les Douze Césars sont traduits en français en 1520, 1530, 1556 et 1641.

${ }^{8}$ Voir Les Vies des hommes illustres [...], édition Jacques Amyot, Paris, Vascosan, 1559, f 464 .

${ }^{9}$ Voir l'édition des Belles-Lettres, t. IX, 1975, introduction et traduction de R. Flacelière et E. Chambry, p. 127.

${ }^{10}$ Voir Plutarque, Vie de Jules César, édition Amyot, p. 000.

${ }^{11}$ Voir l'introduction de l'édition des Belles Lettres, p. 127.
} 
duc»? Plutarque les avait déjà mentionnés $(17,2)$. L'extraordinaire vitesse de déplacement du conquérant de la Gaule ? On la trouve aussi chez lui, de même que les anecdotes révélant sa rapidité d'esprit $(18,7)$. Ce que plus tard Baudelaire appellera son dandysme ${ }^{12}$, est suggéré par Plutarque quand il note qu' "il se grattait la tête d'un seul doigt » pour ne pas déranger la savante ordonnance de ses cheveux qui étaient rares $^{13}$. Suétone de son côté rapporte les rumeurs qui ont couru sur les tendances sodomites de son personnage ${ }^{14}$. Au-delà de ces "petits faits vrais", dont la Renaissance va se montrer friande, les deux biographies antiques, et surtout celle de Plutarque, baignent dans une atmosphère étrange, bien propre à faire rêver les auteurs de la Renaissance. Un exemple suffira, emprunté à Plutarque : "On dit que la nuict de devant qu'il passa ceste riviere [le Rubicon], il eut en dormant une illusion damnable, c'est qu'il luy fut advis qu'il avoit affaire avec sa propre mere $»^{15}$. Suétone rapporte lui aussi le songe en question, mais le place à l'époque où César n'était encore que questeur ${ }^{16}$. Évidemment, un songe de ce genre jette une lumière trouble sur l'entrée du héros, les armes à la main, dans son pays natal. Il place son entreprise sous un jour criminel, d'autant que le biographe ne fait pas état de l'interprétation classique de ce songe : la mère, c'est la terre-patrie ${ }^{17}$.

Le dispositif de Suétone, très lu à la Renaissance, est pourtant différent de celui du biographe grec. Plus de parallèles explicites ou implicites. Il appartiendra au lecteur, s'il le souhaite, de les établir en comparant deux à deux les vies des douze Césars. Mais Suétone est tout aussi curieux de la vie privée de ses personnages et de leur manière de vivre, à laquelle il consacre en général quelques chapitres au moment où la destinée de l'empereur a atteint son point culminant. Dans le cas de César, un portrait «en pied» occupe les chapitres $45-76$, juste avant le récit des Ides de Mars. On a déjà dit que certains de ses traits se trouvaient aussi chez Plutarque. D'autres en revanche sont tout à fait nouveaux, à commencer par les rumeurs de sodomie qui mettent le personnage un peu à part et l'entourent d'une atmosphère sulfureuse. Ce qui le prouve, a contrario, c'est la suppression de ce passage dans un certain nombre d'éditions ${ }^{18}$. Les plus grands admirateurs de César, comme Pétrarque, se garderont bien d'y faire allusion. En revanche, presque toutes les Vies écrites par la Renaissance font un sort à des vers que César, selon Suétone ${ }^{19}$, avait constamment à la bouche dès sa jeunesse. Ils sont empruntés aux Phéniciennes

\footnotetext{
${ }^{12}$ «Le dandysme est une institution vague, aussi bizarre que le duel; très ancienne, puisque César, Catilina, Alcibiade nous en fournissent des types éclatants " (Curiosités esthétiques, Euvres complètes, Gallimard, «La Pléiade », 1951, p. 898).

${ }^{13}$ Plutarque, op. cit, et Suétone, op. cit.

${ }^{14}$ Vie de César, ch. 49.

${ }^{15}$ Plutarque, Vie de Julius Casar, édition Amyot, $\mathrm{f}^{\circ} 503 \mathrm{v}^{\circ}$.

${ }^{16}$ Op. cit, 7, 2.

${ }^{17}$ Voir Marie Delcourt, Edipe ou la légende du conquérant, Paris, Les Belles-Lettres, 1981, p. 202. Selon l'auteur, César ne pouvait être réellement troublé par ce songe car il connaissait l'interprétation classique ainsi que l'oracle donné aux fils de Tarquin et à Brutus par la Pythie de Delphes (Tite-Live, I, 56) : «Le pouvoir suprême appartiendrait à celui [...] qui le premier aura donné un baiser à sa mère. Brutus comprend tout de suite et baise la terre puisqu'elle est la mère de tous » (p. 197).

${ }^{18}$ Par exemple dans la traduction de Du Teil, Des Vies des douze Césars [...], Paris, 1641.

${ }^{19}$ Vie de César, ch. 30.
} 
d'Euripide (v. 524-525) et Cicéron les traduit ainsi : Nam si violandum est jus, regnandi gratia /Violandum est; aliis rebus pietatem colas $^{20}$. Pour Cicéron, le premier à raconter que César les citait souvent, le sens de ces vers est clair : l'ambition de César s'est manifestée dès sa jeunesse, très tôt il aurait aspiré au pouvoir suprême, et pour y parvenir, il était prêt à violer toutes les lois. En d'autres termes, plus proches du vocabulaire politique médiéval, César a été un tyran d'usurpation. De là à faire de Brutus et de Cassius les héros de la liberté, il n'y a qu'un pas que l'on hésite pourtant à franchir ${ }^{21}$.

J'en aurai fini avec l'antiquité et les parallèles qu'elle nous offre lorsque j'aurai cité aussi un passage de Pline l'Ancien, curieusement oublié par les historiens. Il se cache dans le livre VII de l'Histoire Naturelle, consacré à l'anthropologie. Car si l'humanité est faible, comme le dit le célèbre prologue de ce livre, elle enfante aussi des hommes exceptionnels. Pline en trouve trois : César (9194), Pompée (95-99), et Caton (100). Du diptyque cher à la plupart des biographes, on passe donc au triptyque. Mais certains textes de la Renaissance, qui font un sort au passage de Pline, ne retiennent que les figures de César et de Pompée. C'est le cas, par exemple, de Blaise de Vigenère. Que nous dit donc l'auteur de l'Histoire Naturelle au sujet de César : « Je tiens quant à moy Jules Cesar dictateur avoir esté le plus excellent de tous autres, en force et vigueur d'entendement: Je n'entends point icy parler de sa grand'constance et magnanimité de courage, ny de ceste sublimité d'Esprit, capable de tout ce qui est contenu sous le Ciel, mais de certaine vivacité à luy propre et particuliere; de sa promptitude en toutes choses, voltigeante, viste et legiere, comme si elle eust des aisles de feu $»^{22}$. Admirable langage que celui de Pline, sourtout dans la traduction de Vigenère, et qui change du tout au tout le regard que l'on peut porter sur César. Les images du feu et de la légèreté nous suggèrent la présence du génie, et comme une familiarité sublime avec l'éther. Pline ne s'attarde pas beaucoup sur les cadavres des guerres civiles et sur la comptabilité dans laquelle certains auteurs s'étaient engagés ${ }^{23}$. Il est des hommes qui dépassent l'humanité ordinaire, ils sont à leur manière des merveilles de la nature. Au départ, Pline en a compté trois. À l'arrivée, il n'en reste plus qu'un : César.

Je voudrais revenir maintenant sur le portrait politique de Jules César, tel que le dessinent les biographies de la Renaissance. Et tenter d'expliquer pourquoi, pendant plusieurs siècles, ceux qui correspondent en gros au haut Moyen Âge, la question de sa tyrannie ne pouvait se poser. Elle ne se posait pas, me semble-t-il, parce que César ne possédait pas encore une figure vraiment individuelle. Il était d'abord et avant tout le fondateur de l'empire romain, qui devait devenir le Saint Empire. Par conséquent, il était aussi intouchable que l'idée même d'empire. Dans la fixation de cette image, une œuvre a joué un rôle essentiel : la Cité de Dieu de saint Augustin.

\footnotetext{
${ }^{20}$ De Officiis, III, 82.

${ }^{21}$ Voir Shakespeare, Jules César.

${ }^{22}$ Les Commentaires de Jules Cesar, des guerres de la Gaule plus ceux des guerres civiles [...] le tout de la version de Blaise de Vigenère [...], Paris, A. l'Augblier, 1589, f. 400.

${ }^{23}$ Histoire naturelle, VII, 92. Le livre VII de Pline a bénéficié à la Renaissance de plusieurs éditions séparées : voir J. Céard, La Nature et les prodiges, nouvelle édition, Genève, Droz, 1996, note 56, p. 12.
} 
Pour lui, en effet, la mission de l'empire était providentielle : «Après la longue durée des illustres empires de l'Orient, Dieu a voulu qu'il y en eût un aussi en Occident, postérieur chronologiquement, mais plus illustre par l'étendue et la grandeur. Pour réduire le mal accablant de nombreux peuples, il a remis l'empire à de tels hommes, qui par l'honneur, les louanges et la gloire ont pris soin de leur patrie où ils recherchaient la gloire elle-même $»^{24}$. L'idée de domination du monde, même si elle n'est pas d'essence chrétienne, est donc loin d'être moralement condamnable. Elle l'est si peu que, dans le chapitre précédent, saint Augustin cite le fameux passage de l'Énéide ${ }^{25}$, où, par la voix d'Anchise, elle est promise aux Romains. Le Moyen Âge les a repris souvent et on les trouve par exemple dans un passage du De Monarchia de Dante ${ }^{26}$. Évidemment, la figure de César lui-même profite du prestige de la notion d'empire puisque c'est lui qui, d'une manière insensible, l'a mis en place. Au reste, les vertus qui lui appartiennent en propre justifient à elles seules l'admiration de saint Augustin pour qui, dans les derniers temps de la République, il n'y eut que deux grandes figures : Caton, qui a sa préférence, et, juste après, $\mathrm{César}^{27}$.

La lecture de saint Augustin a donc diffusé une image de César étroitement liée à son œuvre: la création de l'empire. Et l'une vaut ce que vaut l'autre. Le besoin de romanesque se fera sentir plus tard, peut-être à l'époque où apparaît le culte des neuf preux. César est l'un d'eux, comme on le sait ${ }^{28}$, mais ce compagnonnage a pour effet de diluer ce qui lui appartient en propre. D'autre part, la noblesse de l'idée d'empire interdit que l'on se pose vraiment, sauf erreur de ma part, la question de la tyrannie. Les lecteurs de Lucain, bien connu du Moyen Âge, ont beau savoir que la personne de la mère-patrie s'est dressée devant lui lors du passage du Rubicon ${ }^{29}$, César échappe à la condamnation. Il faudrait bien entendu de nombreuses enquêtes, que je n'ai pas faites, pour affirmer en toute certitude que César n'est pas considéré comme un tyran pendant toute la durée du Moyen Âge. J'ai trouvé pourtant une confirmation de mon propos dans une Vie de César, toujours manuscrite et qui date du milieu du $X^{\mathrm{e}}$ siècle $^{30}$. Je n'en retiendrai que le passage du Rubicon. L'auteur, anonyme, ne manque évidemment pas la prosopopée de la patrie, trouvée dans la Pharsale ou chez un intermédiaire. Mais elle est suivie d'une scène bien étrange, que l'auteur doit à Suétone. Alors que César, quelque peu troublé par l'apparition de la patrie, hésite à franchir le fleuve fatidique, il voit un géant, assis sur l'un des berges et qui joue divinement d'un chalumeau rustique, charmant de cette façon les pasteurs des alentours ${ }^{31}$. Puis, il pose son instrument de musique et, s'emparant de la trompette d'un soldat de César, il s'avance dans les eaux du Rubicon et invite la

${ }^{24}$ Cité de Dieu, V, 13, traduction Jean-Louis Dumas, Paris, Gallimard, «La Pléiade », 2000, p. 197.

${ }^{25}$ V. 847-853.

${ }^{26}$ II, 6, Euvres complètes, Paris, Gallimard, La Pléiade, 1965, p. 685.

${ }^{27}$ Cité de Dieu, V, 12, édition citée, p. 195.

${ }^{28}$ Voir J. Huizinga, Le Déclin du Moyen Âge, tr. fr., Paris, Payot, 1961, p. 84 et suiv.

${ }^{29}$ La Pharsale, I, v. 183-222.

${ }^{30} \mathrm{BN}$, ms. Fr., 22540. J'ai trouvé cette Histoire de Jules Cesar en feuilletant le catalogue de l'exposition sur Les Manuscrits à peintures, 1440-1520, Paris, Flammarion/Bibliothèque Nationale, 1994, p. 327. Il était signalé en raison de ses enluminures, dues à l'atelier de Jean Colombe.

${ }^{31}$ Manuscrit cité, $\mathrm{f}^{\circ} 161 \mathrm{r}^{\circ}$; Suétone, ch. 32 
troupe à le suivre. César alors se décide. Que représente donc ce géant musicien ? Il est bien difficile de le dire. Une chose est sûre : son intervention contrebalance celle de la Mère-patrie. Une permission succède à une interdiction. S'il incarne les forces de la terre, comme cela est souvent le cas pour les géants, alors il faut dire que celleci épouse le désir de César. Bien entendu, celui-ci ne se réalisera pas sans violence, ce que signifie sans doute le remplacement du chalumeau pastoral par la trompette guerrière. César marche vers le pouvoir personnel, mais avec une mystérieuse et cosmique complicité.

Peu à peu cependant, au fur et mesure que l'idée d'empire s'affaiblit, le visage du tyran apparaitt. On le voit bien dans un ouvrage au reste médiocre, qui compile les faits et gestes des empereurs de Rome: celui du Vénitien Jean-Baptiste Egnazio, de son vrai nom Cipelli, paru chez Alde en 1516 et publié en français par Geoffroy Tory en $1529^{32}$. La première phrase se passe presque de commentaire : «Le premier cy nommé Jules César s'attribua par force la Dictature, c'est-à-dire la Souveraine Authorité et Domination sur tous les Romains». Suit un bref résumé des combats de César. Et voici comment se termine la notice : «Il estoit prince tresvertueux, tant en temps de paix que de guerre, sur tous clement et de bon vouloir. S'il eust [équivalent de l'utinam latin] plustost aymé deffendre la liberté de son pays que l'opprimer ${ }^{33}$. Notons au passage que nous devons ce jugement à un Vénitien, et que Venise, traditionnellement, est jalouse de sa liberté et méfiante à l'égard des grandes puissances. Il n'empêche : le ton est donné. On le retrouve par la suite chez les auteurs de Vies, par exemple chez Du Verdier, dans sa Prosopographie, mais avec des inflexions significatives qui atténuent les griefs: «Apres tous ces triomphes, les Romains faisans joug à l'heur de Cæsar et recevans le mors en la bouche en intention et esperance que la domination d'un seul leur apporteroit quelque relasche de tant de calamitez, que les guerres civiles leur avoyent faict souffrir, l'esleverent et establirent Dictateur perpetuel pour le temps de sa vie : ce qui estoit evidemment une tyrannie certaine, pour autant qu'ils adjoustoyent à sa souveraine auctorité et au plain pouvoir de la Dictature l'asseurance de n'en estre jamais desmis $\aleph^{34}$. Vous aurez remarqué comme moi que Du Verdier, s'il parle bien de tyrannie, donne à celle-ci des circonstances atténuantes. C'est une cité épuisée par les guerres civiles qui a confié son sort à César. On trouverait presque ici un écho de la fameuse servitude volontaire. Au fond, César n'a pas obligé les Romains à lui donner ce titre de dictateur. Un petit mot doit aussi retenir l'attention : celui d' «heur». Il signifie en somme que pendant ses campagnes militaires, César a été le protégé des dieux, il a été felix. C'est à ce titre aussi qu'il a envoûté les Romains.

Plusieurs biographes de la Renaissance rapportent le goût de César pour les vers d'Euripide que j'ai cités tout à l'heure. Beaucoup savent aussi que l'informateur de Suétone n'est autre que Cicéron lui-même, qui dénonce dans le De Officiis l'appétit tyrannique de César. Le commentaire de Béroalde ne semble pas autrement gêné par cette maxime. Il explique pédagogiquement que, chez Euripide, ces vers

\footnotetext{
${ }^{32}$ Sommaire de chroniques contenans les Vies, Gestes, et cas fortuitz de tous les Empereurs depuis Jules César [...], Paris, [G. Tory], 1529, seconde édition en 1543. Sur cet auteur, voir le Dizionario biografico degli Italiani, t. XXV, p. 698-702.

${ }^{33}$ Op. cit., f ${ }^{\circ} 1$.

${ }^{34}$ Prosopographie [...], Lyon, P. Frelon, 1604, p. 745.
} 
sont placés dans la bouche d'Étéocle, cupidum dominationis, et que César a voulu dire : Si injuriari oportet, super Tyrannide pulcherrimum est injuriari. In aliis pietatem colere est oportunum ${ }^{35}$. De la part d'un humaniste chrétien, on aurait pu attendre quelques réserves. L'embarras des humanistes s'explique peut-être par l'histoire de ces deux vers d'Euripide. Ils se trouvent sans doute dans le texte du De Officiis. Mais ils se lisent aussi dans le Florilège de Stobée, dont personne ne néglige les bons offices, et plus précisément dans la section qui a pour titre, dans l'édition Gesner: Quod optimum sit monarchia ${ }^{36}$. Qui plus est, la citation d'Euripide se trouve juste après les vers de l'Iliade (II, 204) sur l'excellence du gouvernement monarchique, vers rendus fameux par le coup d'envoi de la Servitude volontaire. Du même coup, il n'est pas facile de distinguer le juste gouvernement d'un monarque de l'appétit de pouvoir d'un tyran, qui lui aussi gouverne seul. On le pourrait peut-être si l'on remontait un peu plus haut dans les vers d'Euripide, à ces vers fiévreux où Étéocle exprime un désir de pouvoir qui s'étend au cosmos. Voilà bien l'hybris du tyran. Les biographes de César, trop respectueux de leur personnage, ne s'y risquent pas. Mais un auteur connaît bien ce passage: Shakespeare. Ce sont ces vers qu'il prête à Hotspur, ou si vous préférez à Henry Percy, dans un passage du premier Henry $I V$ : «Par le ciel! Je serais tenté de m'élancer jusqu'à la pâle face de la lune pour en arracher l'Honneur éclatant ou de plonger dans les abîmes de l'Océan, jusqu'à des profondeurs restées inaccessibles à la sonde pour en retirer par les cheveux l'Honneur englouti, si le Libérateur pouvait recueillir, seul et sans rival, toute la gloire de son action. Mais foin d'une médaille partagée ${ }^{37}$. Comment lire ce passage ? Comme une furieuse apologie de la soif de pouvoir? Ou comme un éloge de l'esprit chevaleresque, tout à fait à sa place dans la bouche du jeune Northumberland, révolté contre le roi en place ? Il est bien difficile de le dire. De la même façon, il est difficile de dire si César est blâmable d'avoir conquis le pouvoir les armes à la main, ou s'il est digne d'éloge pour avoir aspiré de toutes ses forces à l'honneur, pour lui-même et pour Rome. Complexité du personnage de César, à travers même les vers qu'il aimait citer. Elle atteint son paroxysme dans la biographie que lui consacre Pétrarque.

L'histoire de ce texte est elle-même assez compliquée ${ }^{38}$. Beaucoup d'incertitudes demeurent quant à la date où l'ouvrage a été entrepris et la chronologie de sa rédaction. On a souvent écrit que Pétrarque y travaillait encore le jour de sa

\footnotetext{
${ }^{35}$ Suétone, De XII Caesaribus libri VIII, Paris, H. Drouart, 1610 avec, parmi d'autres, le commentaire de Philippe Béroalde, col. 53 ; pour prévenir toute confusion, précisons qu'il s'agit ici de Philippe Béroalde l'Ancien, 1453-1505. Voir la notice des Centuria latina, Genève, Droz, 1997, col. 109-121.

${ }^{36}$ Ioannis Stobei sententice [...], éd. C. Gesner, Zurich, 1542, Sermo XLV, p. 214.

${ }^{37}$ Shakespeare, Euvres complètes, édition citée, t. I, p. 616.

${ }^{38}$ Voir la notice de Léon Dorez, en tête de la reproduction phototypique du manuscrit latin 5784, BN, Département des manuscrits, Fac simile 245 ; Pierre de Nolhac, «Le De viris illustribus de Pétrarque » (notice sur les manuscrits originaux, suivie de fragments inédits), in Notices et extraits des manuscrits, t. XXXIV, $1^{\text {ere }}$ partie, 1891, p. 12-15; et surtout l'introduction de Giuliana Crevatin à sa récente édition du De gestis Cesaris, Pise, École Normale Supérieure, 2003. Le texte de Pétrarque sera cité d'après cette édition.
} 
mort. L'examen du manuscrit qui fait autoritée ${ }^{39}$, et qui comporte d'assez nombreuses ratures et additions ainsi que celui de l'écriture, qui se modifie au cours des années ${ }^{40}$, semble indiquer à la fois que l'auteur lui accordait une grande importance et que, d'autre part, le De gestis Cesaris était devenu peu à peu un ouvrage très personnel ${ }^{41}$.

Pétrarque ne cache pas du tout l'ambition de son personnage. À son tour, il raconte par exemple le dépit qu'il éprouve devant la statue d'Alexandre qui, au même âge, avait déjà conquis la moitié du monde ${ }^{42}$. Il ne cache pas davantage que son héros avait toujours à la bouche (semper in ore habuisse) les vers d'Euripide dont il a été question plus haut ${ }^{43}$. Mais il rappelle au passage que Cicéron, à la source de cette information, a été d'abord l'ami, puis l'ennemi juré de César: manière de jeter un doute sur la véracité de son témoignage. Pour le corriger, Pétrarque cite souvent les lettres où César proclame son attachement à la paix ${ }^{44}$, alors même qu'il est engagé dans la lutte contre Pompée. Il est sûr que, la veille même de la bataille de Pharsale, il essaie encore de négocier avec son adversaire ${ }^{45}$. Nous savons fort bien, après les dictatures du $\mathrm{XX}^{\mathrm{e}}$, ce que vaut ce genre de discours sur l'amour de la paix, spécialité de tous les despotes. Ce qui me paraît intéressant dans la biographie écrite par Pétrarque, c'est qu'il n'est pas naîf, et ne cherche pas à innocenter son héros. Il sait se montrer fort sévère à son sujet, écrivant par exemple: Nulla sufficiens causa est contra patriam arma moventibus ${ }^{46}$. César, plus d'une fois, a passé la mesure, sa route triomphale est jonchée de centaines de milliers de morts ${ }^{47}$. Il n'est donc pas un personnage tout d'une pièce. Le meilleur moyen de s'en rendre compte est de jeter un coup d'œil sur la scène à faire, celle du passage du Rubicon ${ }^{48}$. Une surprise nous attend : la célèbre prosopopée de la patrie a disparu. L'auteur n'en a pas besoin pour nous rendre sensibles à la perplexité de César, tacite examinans le pour et le contre du franchissement du fleuve. La nuit est tombée, le fond de la vallée est à l'image de l'âme du conquérant: perplexus, enchevêtré. Alors que César hésite, hæerens dubiusque animi, voici que lui apparaît l'étrange géant dont j'ai parlé tout à l'heure et qui joue du chalumeau. Pas pour longtemps, on le sait, puisqu'il s'empare de la trompette d'un soldat et se met à en sonner en traversant le fleuve.

\footnotetext{
39 Bibliothèque Nationale, ms. Lat., 5784. Voir l'étude codicologique très précise de G. Crevatin, édition citée, p. XVII-LXI.

${ }^{40}$ Voir A. Petrucci, La scrittura di Francesco Petrarca, Cité du Vatican, 1967.

${ }^{41}$ Pétrarque, rappelons-le, est mort en 1374. Pour ce qui concerne le De viris illustribus, entrepris vers 1340, voir l'édition critique de G. Martellotti, Florence, Sansoni, 1964. Le De gestis Cesaris s'inscrit tout à fait dans un mouvement de "renouveau historiographique » auquel appartient également, en Italie, Benvenuto da Imola, l'auteur du Romuleon, écrit entre 1361 et 1364 : voir l'édition de la traduction médiévale de ce texte par Frédéric Duval, Genève, Droz, 2000, p. 12 et, du même auteur, La traduction du Romuleon par Sébastien Mamerot. Étude sur la diffusion de l'histoire romaine en langue vernaculaire à la fin du Moyen Âge, Genève, Droz, 2001.

${ }^{42}$ De gestis Cesaris, édition citée, p. 4.

${ }^{43}$ Ibid., p. 207.

${ }^{44}$ Ibid., pp. 207, 211, 214.

${ }^{45}$ Ibid., p. 237.

${ }^{46}$ Ibid., p. 199.

${ }^{47}$ Ibid., p. 301.

${ }^{48}$ Ibid., p. 210-211.
} 
César alors se décide : Eatur, ait, quo deorum prodigia atque hostium vocat iniqui$\operatorname{tas}^{49}$. Analysons un peu. César est entouré de prodiges, les dieux lui adressent des signes. Ils semblent lui dire que le temps de la paix, symbolisé par le chalumeau rustique, est fini. C'est maintenant celui de la guerre, rendue inévitable par les adversaires de César. Est-elle pour autant juste? On peut en douter. C'est un bien étrange guide que ce géant musicien. Mais Pétrarque a su mieux que quiconque mettre en scène la perplexité de son personnage. Va-t-on s'en étonner? Certainement pas : qui a été plus perplexe que Pétrarque lui-même?

D'un bout à l'autre de cette œuvre, il orchestre d'autre part le thème de l'invidia. César, dès le début de sa vie, a été la victime de la jalousie, de l'envie. Tel est le lot de ceux qui cherchent la gloire. Il aurait pu sans doute faire le choix de Thersite $^{50}$ ou celui de Scipion qui s'y est soustrait grâce à l'exil ${ }^{51}$. Ce ne fut pas le choix de César. Ses ennemis furent même si machiavéliques qu'ils voulurent pour lui plus d'honneurs que ses amis : c'était en effet la meilleure manière de le rendre odieux au plus grand nombre. En fin de compte, c'est l'invidia qui est la cause des Ides de Mars. Pétrarque ironise amèrement sur toutes les raisons qu'on a données pour expliquer l'assassinat de son grand homme : l'histoire de la couronne ou celle de l'affront infligé à une délégation de sénateurs venue lui rendre visite et devant laquelle César néglige de se lever ${ }^{52}$. Tout cela ne pèse pas lourd dans le grand combat qui oppose et opposera toujours la grandeur à la médiocrité. Pétrarque croit d'ailleurs savoir que le lieu où il fut assassiné était proche de la Roche tarpéienne ${ }^{53}$. Parvenu au faîte de sa grandeur, César devait tomber, selon un schéma bien mis à jour par Patricia Eichel-Lojkine dans son livre ${ }^{54}$. Derrière les mesquines ambitions des adversaires de César, se tient sans doute la Fortune elle-même. Nous sommes déjà en pleine Renaissance comme le prouve l'omniprésence de la figure de l'invidia chez Boccace ${ }^{55}$.

Peu à peu se dessine la figure d'un personnage d'exception, assez proche du portrait que trace de lui Pline l'Ancien. De toute évidence, Pétrarque est fasciné par cet être de mouvement, aussi rapide dans ses actes que dans l'exercice de la pensée. Il se plaît à toutes les anecdotes qui l'illustrent. Son héros est capable de dicter plusieurs lettres en même temps ${ }^{56}$. Il arrive parfois avant les messagers qu'il a dépê-

\footnotetext{
${ }^{49}$ Ibid., p. 211. La source se trouve dans Suétone, Vie de César, ch. 32. Elle est reprise presque au même moment dans le Romuleon: voir la traduction de Sébastien Mamerot, édition citée, p. 169. La parenté entre les deux textes est tout à fait troublante : lequel imite l'autre ?

${ }^{50}$ Ibid., p. 199. D'ordinaire, Thersite (Iliade, II, 212 et suiv.) est une figure de la laideur. Il devient chez Pétrarque celle de l'obscurité médiocre.

${ }^{51}$ Faut-il rappeler que Pétrarque a chanté Scipion l'Africain dans son grand poème latin: l'Africa, commencé à Avignon en 1338 ? Scipion l'Africain est d'ailleurs également présent dans le De viris illustribus, commencé à peu près au même moment.

${ }^{52}$ Ibid., p. 218.

${ }^{53}$ Ibid., p. 331.

${ }^{54}$ Le Siècle des grands hommes, op. cit., p. 159-173.

${ }^{55}$ Dans le De casibus, mais encore plus dans la lettre de consolation à Pino dei Rossi : Boccace, Opera, édition Mondadori, p. 646. Voir aussi André Thevet, Les Vrais pourtraicts et Vies des hommes illustres [...], Paris, 1584, p. 624.

${ }^{56}$ De gestis Cesaris, p. 306.
} 
chés : Celeritatis non tantum mire sed incredibilis fuit ${ }^{57}$. Il écrit ou dicte en traversant les Alpes. Il possède en somme la vitesse du feu, l'aisance du génie, capable de faire plusieurs choses à la fois. C'est bien ce que Pline avait dit, dans la belle traduction de Vigenère, quand il célébrait cette promptitude «voltigeante, viste et legiere comme si elle eust des aisles de feu ». Pétrarque connaissait bien entendu le passage éclatant du Paradis de Dante sur la vitesse de l'aigle romaine, conduite par César : «Son vol, après qu'il sortit de Ravenne/ Pour sauter Rubicon, prit tel essor/ Que plume ou langue à le suivre s'épuise ${ }^{58}{ }^{5} \mathrm{~N}$ 'y aurait-il donc aucune ombre dans les dons de César? Si, bien sûr, et tous les biographes mentionnent les fameux accès d'épilepsie, ou, pour parler comme le XVI siècle, le «mal caduc» dont il souffrait. Pétrarque ne le passe pas sous silence: Tamen et animo nonnunquam linqui solitus et per quietem visis concuti ${ }^{59}$. Cette phrase mérite une seconde d'attention. D'abord parce que l'auteur ne désigne pas l'épilepsie par le terme médical classique à l'époque: comitialis morbus ${ }^{60}$. L'expression dont il se sert (animo nonnunquam linqui) assimile l'épilepsie à une perte de connaissance. D'autre part, dans cette même phrase, Pétrarque affirme que César est sujet aux songes ou aux visions. Avant que la philosophie de Ficin ne s'en mêle ${ }^{61}$, c'est une manière de suggérer que la maladie de César le met en communication avec les dieux, que la perte de connaissance est le prélude à d'autres connaissances. Elle possède la noblesse que les mentalités populaires, à la fois séduites et effrayées, concèdent au «haut mal». D'une manière ou d'une autre, César a commerce avec la transcendance.

Avançons encore un peu dans ce portrait de César qui, au début, ne devait être que l'un des hommes illustres. Pétrarque conclut une anecdote où il est aussi question d'Alexandre en écrivant ceci : Profecto, ut in multis in hoc quoque unicus Casar est ${ }^{62}$. Le support de cette réflexion est mince, mais elle va loin. D'autant qu'elle trouve d'autres échos dans le texte. C'est par exemple César lui-même qui déclare: Nihil enim malo quam et me mei similem esse $e^{63}$, propos qui suscite l'enthousiasme de son biographe: Quo dicto vix aliquid unquam dici gloriosius ac magnificentius audivi ${ }^{64}$. César est magnifique en proclamant que sa règle est de ressembler à lui-même. Telle est la forme que prend chez lui le culte du moi. Il est déjà un héros de la Renaissance, telle du moins que je la vois, traçant une voie solitaire et proprement magnifique. Mais du même coup, il échappe à l'imitation et à la straté-

\footnotetext{
${ }^{57}$ Ibid., p. 307.

${ }^{58}$ La Divine Comédie, Le Paradis, VI, 61-63, traduction André Pézard, Paris, Gallimard, «La Pléiade », 1965, p. 406.

${ }^{59}$ De gestis Cesaris, p. 304.

${ }^{60}$ "Comitialis », parce que, à Rome, les comices ne pouvaient se réunir lorsque quelqu'un était touché par une crise d'épilepsie.

${ }^{61}$ Voir la Théologie platonicienne de l'immortalité de l'âme, XIII, 2, édition R. Marcel, Paris, Les Belles Lettres, 1964, t. II, p. 119. Précisons cependant que Ficin, dans ce passage, ne parle pas des syncopes dues à l'épilepsie.

${ }^{62}$ De gestis Cesaris, p. 244.

${ }^{63}$ Ibid., p. 217. Voir Cicéron, Att, IX, 16, 1-2.

${ }^{64}$ Ibid.
} 
gie de l'exemplum, chère au Moyen Âge ${ }^{65}$. Puisque César n'a imité personne, sauf peut-être Alexandre, il ne pourra être imité de personne. Il est vraiment unique. On comprend alors pourquoi il est sorti du cadre vertueux du De viris illustribus. Si, comme l'a écrit Pierre Maréchaux ${ }^{66}$, Pétrarque proposait en chacun de ceux-ci une grande vertu à imiter, son projet s'effondre lorsqu'il s'agit de César. On ne peut imiter ce qui est unique.

Nous touchons là aux aspects les plus mystérieux, et, d'une certaine façon, les plus déconcertants de la biographie de Pétrarque. Plus d'un passage de cette œuvre possède en effet des résonances religieuses, et même christiques. La rapidité de déplacement de César, sa faculté d'apparaître là où on ne l'attend pas possède déjà quelque analogie avec certaines figures des mythes religieux. Il y a plus, beaucoup plus. Ce passage d'abord, qui se situe au moment où César poursuit Pompée en Grèce. Il a déjà traversé le bras de mer qui sépare l'Italie de l'Épire, lorsqu'il se rend compte qu'une fois de plus il est allé trop vite et que le gros des troupes n'a pas suivi. Il décide alors de retraverser la mer dans l'autre sens, vers l'Italie. Une tempête survient, qui l'empêche d'aborder. Le pilote prend peur. Et voici ce qu'on lit: Nota est vox ejus ad exterritum nocturno equoreoque periculo gubernatorem: Quid te metuis ? Casarem vehis ${ }^{67}$. Comment ne pas penser à l'épisode de la tempête apaisée raconté dans les Évangiles ${ }^{68}$, aux propres paroles du Christ demandant à ses disciples de ne pas avoir peur. On me dira que cet épisode se trouve déjà chez $\mathrm{Plu-}$ tarque, qui ne songeait pas à l'Évangile ${ }^{69}$. Soit. Mais Pétrarque, lui, y songeait forcément. Le détail de l'imitation montre d'ailleurs qu'il a travaillé le texte de l'auteur grec pour renforcer le sens du mystère. C'est l'effet produit par la voix qui est souligné plutôt que l'énonciation de la parole. Chez Plutarque, d'autre part, César prenait la main du pilote, familiarité exclue du texte de Pétrarque. La teneur même de son propos est assez différente, comme on peut s'en rendre compte en citant la traduction d'Amyot, très fidèle à l'auteur grec: «Mon amy, aies bon courage, et poulse hardiment sans craindre rien, car tu menes Cæsar et sa fortune quand et luy ${ }^{70}$. Le terme «fortune», sans doute trop païen, a disparu. En cinq mots, pas un de plus, César donne une leçon de courage et de brevitas stylistique. On ne doit pas avoir peur quand on se trouve dans sa compagnie. C'est tout juste si Pétrarque ne dit pas qu'il commande aux éléments. On pourrait citer bien d'autres passages possédant des connotations religieuses. L'autre grand moment évangélique et même christique se trouve bien entendu dans le récit des Ides de Mars. Parce que César s'avance vers la mort comme le Christ s'avance vers la sienne, dédaignant les avertissements

\footnotetext{
${ }^{65}$ Notons d'ailleurs que la figure de César apparaît peu dans les exempla médiévaux, si du moins on en croit J. Th. Welter, L'Exemple dans la littérature du Moyen Âge, Paris-Toulouse, 1927.

${ }^{66}$ Centuria latina, notice « Pétrarque », Genève, Droz, 1997, p. 608.

${ }^{67}$ De gestis Cesaris, p. 228.

${ }^{68} \mathrm{Mt}, 14,22-33, \mathrm{Mc}, 6,45-52, \mathrm{Jn}, 6,16-21$.

${ }^{69}$ Sans doute. N'oublions pas cependant que, pour Montaigne, Plutarque est un auteur très religieux : Essais, II, 12, édition Villey-Saulnier, p. 603 ; et que le célèbre épisode du grand Pan (Rabelais, Quart Livre, ch. XXVIII) est emprunté à un opuscule de Plutarque Sur la cessation des oracles. On pourrait facilement allonger la liste des œuvres de Plutarque susceptibles d'une lecture chrétienne.

${ }^{70}$ Op. cit., $\mathrm{f}^{\circ} 505 \mathrm{r}^{\circ}$.
} 
qu'on lui donne $^{71}$, ce qui a fait dire à certains auteurs, cités par le nôtre: Quidam opinantur Casarem et mortem suam prescisse, et sic mori voluisse ${ }^{72}$. Autre analogie : les prodiges qui précèdent ou suivent la mort du fils de Dieu et celle du premier empereur. «Vous allez un peu loin», me dira-t-on. Je ne crois pas. En tout cas, avant Pétrarque, Dante est allé aussi loin. C'est lui qui, dans l'Enfer, rapproche le supplice de Judas Iscariote, celui qui a trahi Jésus, de ceux de Brutus et Cassius, les meurtriers de César ${ }^{73}$. Plus énigmatique est la référence au deuil des Juifs de Rome qui sepulcrum ejus aliquot continuis noctibus pio officio frequentarunt ${ }^{74}$. César avait-il donc des titres à leur reconnaissance? Le Romuleon en françois en parle également : «En cestui souverain et publicque plaint et pleur fit la multitude des gens estranges lamentacion selon la coustume et especialment les juyfs qui par continuelles nuyts visitoyent sa sepulture $»^{75}$. Risquons une hypothèse : ce deuil des Juifs donne une note religieuse aux funérailles de César. Il signifie que la mort de César a touché des hommes tout à fait étrangers à la religion romaine et à toutes les apothéoses.

On pourrait poursuivre l'analyse de ce texte étonnant. Une chose me paraît sûre : Pétrarque n'a pas écrit cette biographie de César en se tenant loin de son objet. Elle possède un frémissement qui en dit long sur la fascination exercée sur lui par cette grande figure. Certains critiques se sont étonnés qu'il soit passé de l'admiration pour Scipion au culte de César ${ }^{76}$. On en a le droit à condition que cette mutation ne soit pas assimilée à un revirement politique, à je ne sais quel passage du mythe républicain au mythe césarien. N'étant pas spécialiste de Pétrarque, j'ai tenté seulement de montrer que la figure de César, telle qu'il la dessine appartient déjà à la Renaissance, et à son culte des grands hommes.

Il y a loin entre la sèche notice biographique du vénitien Ignazio et le somptueux De gestis Cesaris de Pétrarque. Et pourtant, cet ouvrage est largement antérieur au premier. Mais le projet n'est pas le même. Sauf à composer un ouvrage monstrueux et qui occuperait plusieurs in-folio, les auteurs de biographies sont obligés de se limiter. César prend place dans un ensemble où se succèdent les empereurs romains, ceux de Byzance, et ceux du saint Empire romain germanique. Ou bien, il voisine avec les hommes illustres de tous les temps et de tous les pays. Il mérite, selon les cas, une ou dix pages, guère plus. Sa personne disparaît derrière sa fonction, éminente sans doute, surtout pour les auteurs du haut Moyen Âge : c'est lui qui a fait naître l'empire romain, qui a fait progresser la pax romana sur la surface de la terre, avant qu'Auguste ne l'achève, permettant au Christ de naître dans un monde

\footnotetext{
${ }^{71}$ Sur l'usage dramatique de ces avertissements, voir Shakespeare, Jules César, II, 2.

${ }^{72}$ Pétrarque, De gestis Cesaris, p. 317.

${ }^{73}$ Enfer, XXXIV, v. 61-65. Voir la note d'André Pézard qui explique que l'une des raisons de ce rapprochement se trouve dans le nom des coupables.

${ }^{74}$ De gestis Cesaris, p. 320. La source (Suétone, ch. 84) n'explique pas grand chose, de même que la note de l'édition Budé : les Juifs de Rome auraient su gré à César de sa victoire sur Pompée qui avait pris Jérusalem.

${ }^{75}$ Édition citée, p. 277.

${ }^{76}$ Voir Pierre Blanc, « De la transgression comme scandale à la transgression comme idéal : la double image de César dans l'œuvre et la pensée de Pétrarque », Présence de César, op. cit., p. $35-55$.
} 
pacifié (ou presque). Face à ce privilège inouï donné par la Providence, que valent les accusations de tyrannie? Et les vers d'Euripide qu'il aurait eus toujours à la bouche ? $^{77}$ Presque rien. Il faudra toute l'audace des monarchomaques, qui ne sont pas de mon ressort, pour que César perde son auréole, et pour que, à certaines conditions, le tyrannicide soit permis. Même quand l'idée d'empire s'affaiblit, César continue à fasciner. Il est insaisissable. Comment cerner la figure de ce dandy, à la fois soigneux de son apparence et d'une résistance physique à toute épreuve ? Sachant parfaitement combattre et parfaitement écrire ? Désirant farouchement le pouvoir et s'avançant vers la mort avec le calme d'un stoïcien à moins que ce ne soit avec la sérénité du Christ? Il n'est même plus un héros, si le propre de celui-ci est de déclencher un mouvement d'imitation. Un mot de Pétrarque résume tout: Cassar unicus.

Daniel Ménager

Paris X-Nanterre.

${ }^{77}$ André Thevet, par exemple, les cite en passant : Les Vrais Pourtraicts et vies des hommes illustres [...], Paris, 1584, p. 622. 